\title{
Public attitudes to, and behaviours taken during, hot weather by vulnerable groups: results from a national survey in England
}

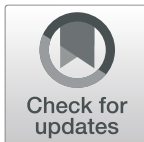

\author{
Bob Erens ${ }^{1 *}$ (D), Lorraine Williams ${ }^{1}$, Josephine Exley ${ }^{1}$, Stefanie Ettelt ${ }^{1}$, Tommaso Manacorda ${ }^{1}$, Shakoor Hajat ${ }^{2}$ and \\ Nicholas Mays ${ }^{1}$
}

\begin{abstract}
Background: Hot weather leads to increased illness and deaths. The Heatwave Plan for England (HWP) aims to protect the population by raising awareness of the dangers of hot weather, especially for those most vulnerable. Individuals at increased risk to the effects of heat include older adults, particularly $75+$, and those with specific chronic conditions, such as diabetes, respiratory and heart conditions. The HWP recommends specific protective actions which relate to five heat-health alert levels (levels $0-4$ ). This study examines the attitudes to hot weather of adults in England, and the protective measures taken during a heatwave.

Methods: As part of a wider evaluation of the implementation and effects of the HWP, a survey $(n=3153)$ and focus groups, a form of group interview facilitated by a researcher, were carried out after the June 2017 level 3 heat-health alert. Survey respondents were categorised into three groups based on their age and health status: 'vulnerable' (aged 75+), 'potentially vulnerable' (aged 18-74 in poor health) and 'not vulnerable' (rest of the adult population) to hot weather. Multivariable logistic regression models identified factors associated with these groups taking protective measures. In-person group discussion, focused on heat-health, were carried out with 25 people, mostly aged $75+$.

Results: Most vulnerable and potentially vulnerable adults do not consider themselves at risk of hot weather and are unaware of the effectiveness of important protective behaviours. Only one-quarter of (potentially) vulnerable adults reported changing their behaviour as a result of hearing hot weather-related health advice during the level 3 alert period. Focus group findings showed many vulnerable adults were more concerned about the effects of the sun's ultra-violet radiation on the skin than on the effects of hot temperatures on health.

Conclusions: Current public health messages appear to be insufficient, given the low level of (potentially) vulnerable adults changing their behaviour during hot weather. In the context of increasingly warmer summers in England due to climate change, public health messaging needs to convince (potentially) vulnerable adults of all the risks of hot weather (not just effects of sunlight on the skin) and of the importance of heat protective measures.
\end{abstract}

Keywords: Heat, Hot weather, Older people, Emergency planning

\footnotetext{
* Correspondence: bob.erens@lshtm.ac.uk

'Policy Innovation \& Evaluation Research Unit, Department of Health Services Research \& Policy, London School of Hygiene \& Tropical Medicine, London WC1H 9SH, UK

Full list of author information is available at the end of the article
}

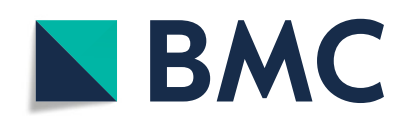

(c) The Author(s). 2021 Open Access This article is licensed under a Creative Commons Attribution 4.0 International License, which permits use, sharing, adaptation, distribution and reproduction in any medium or format, as long as you give appropriate credit to the original author(s) and the source, provide a link to the Creative Commons licence, and indicate if changes were made. The images or other third party material in this article are included in the article's Creative Commons licence, unless indicated otherwise in a credit line to the material. If material is not included in the article's Creative Commons licence and your intended use is not permitted by statutory regulation or exceeds the permitted use, you will need to obtain permission directly from the copyright holder. To view a copy of this licence, visit http://creativecommons.org/licenses/by/4.0/. The Creative Commons Public Domain Dedication waiver (http://creativecommons.org/publicdomain/zero/1.0/) applies to the data made available in this article, unless otherwise stated in a credit line to the data. 


\section{Background}

In England, periods of hot weather lead to increases in deaths and illness [1]. A severe heatwave in 2003 led to over 2000 excess deaths in England and Wales, especially in southern regions and among people aged 75+ [2]. With predictions of more frequent and increasingly hotter summers in England [3], accompanied by increasing numbers of people aged 75+, awareness of heat protection behaviours is of growing importance.

Since 2004, the government has implemented a Heatwave Plan for England (HWP) [4], which aims to protect the population by raising awareness of the dangers of severe heat, and recommends protective actions to be taken by National Health Service (NHS), local government, community groups and individuals [5]. The specific actions to be taken relate to five heat-health alert levels (levels 0-4). Levels 3 and 4 are triggered when specified temperature thresholds are reached. The temperature thresholds vary by region within England, but average to $30 \mathrm{C}$ by day and $15 \mathrm{C}$ overnight for at least two consecutive days. Additional file 1 shows the temperature thresholds for level 3 alerts for each region in the country. In 2017 a level 3 alert was issued for 3 days in June, and in 2018 there were three separate level 3 alerts issued, over July and August, lasting for seven, five and 1 day respectively.

Understanding risk, and how to adapt individual behaviours to prevent illness or death during hot weather, is especially important for those most at-risk to the effects of heat [6]. The HWP identifies factors that increase an individual's risk during hot weather. These include age, particularly $75+$, as ageing diminishes thermoregulatory function [7]; the very young; chronic or severe illness e.g. certain respiratory and heart conditions, diabetes; alcohol and/or drug dependency; homelessness; inability to adapt behaviour to keeping cool; and environmental factors (e.g. living in urban areas).

Results from surveys after level 3 heat-health alerts in England in 2013 showed that, despite widespread awareness of the effectiveness of most protective behaviours, many adults did not consider themselves to be at-risk and relatively few changed their behaviour during the hot weather $[8,9]$. These findings may be partly explained by the positive feelings many UK residents hold towards warm summers, which tend to reduce perceptions of risk [10].

In 2016, the Department of Health and Social Care (DHSC) commissioned an independent evaluation of the implementation and effects of the HWP [11]. This paper presents findings from a survey and focus groups undertaken in England in 2017 and 2018 to examine the protective measures taken by the population in response to a specific heat-health alert in June 2017. It compares perceptions of the effectiveness of heat protection measures among three groups: older people aged 75+ (vulnerable group); adults aged $18-74$ in poor health (potentially vulnerable group); and the rest of the adult population (not vulnerable). It also examines behaviour change during the level 3 heat-health alert between 16 and 23 June, which was associated with an estimated 666 excess deaths [12].

\section{Methods \\ Survey \\ Survey development}

The survey, modelled on two earlier surveys [8, 10], included questions on: whether respondents love hot weather and perceive it as a risk to their health (rated on a 5-point scale from strongly agree to strongly disagree); and perceived effectiveness of nine heat protection measures (rated on a 5-point scale from completely effective to not at all effective).

Individuals who were in England during the level 3 heat-health alert in June 2017 were asked about their experiences, including whether: they had heard heat-alerts and health advice; they had changed their behaviour as a result; and taken any heat protection measures (rated on a 5-point scale from never to always). Respondents were asked whether they had experienced any health effects as a result of hot weather. The survey questionnaire is shown in Additional File 2.

The questions were included in a larger survey conducted by the National Centre for Social Research (NatCen), which collected demographic data and questions on self-rated health (on a 5-point scale from very good to very bad) and whether respondents had any longstanding (i.e. anything that had lasted at least 12 months) physical or mental health condition that limited their ability to carry out normal activities.

\section{Data collection}

NatCen conducted the survey between 24 August - 24 September 2017 using its panel, which is representative of the population aged $18+$ living at private residential addresses in England.

Participants were recruited using a sequential mixed mode design, with panel members first invited to complete the questionnaire online. Those who had not completed the survey online after 2 weeks, including those without internet access or those with language or literacy problems, were contacted by telephone to complete an interview. Respondents were sent a $£ 5$ gift card as a 'thank you', which is standard practice for surveys using the NatCen panel.

Of the 3153 panel members invited to participate, the achieved sample included 1633 online interviews and 245 telephone interviews, giving a response rate of $60 \%$. 
Further details of the NatCen panel can be found in Jessop [13].

\section{Analysis}

Respondents' vulnerability to hot weather was estimated based on their age, self-reported health status and whether they had a limiting long-standing illness (LLSI). Respondents were classified into three groups: (1) 'not vulnerable' (aged 18-74 with no underlying health condition); (2) 'potentially vulnerable' (aged 18-74 with an LLSI and/or in bad/very bad health); and (3) 'vulnerable' (aged 75+). Six respondents with insufficient information were excluded. Data were analysed in STATA (version 16.1).

Non-response weights were calculated to minimise any bias introduced by differential response among population sub-groups [13]. For each variable, percent frequencies and $95 \%$ confidence intervals (CIs) were calculated, weighted for non-response using the survey (svyset and svy) commands. Finally, multivariable logistic regression models were used to examine factors associated with vulnerable and potentially vulnerable individuals taking heat protection behaviours. Each heat protection behaviour was dichotomised as always/often versus occasionally/rarely/never. We present odds ratios (ORs) based on modelling each behaviour in a separate logistic regression model, which included: age; health (good/very good, fair or bad/very bad); LLSI (none, does not affect day-to-day life or affects day-to-day life); household type (living with someone or living alone); respondent's perception of the effectiveness of the measure (completely/very effective or not/slightly/somewhat effective); perceived risk of hot weather to respondent's health (strongly agree/agree, neutral or strongly disagree/disagree); whether respondent loved hot weather (strongly agree/agree, neutral or strongly disagree/disagree); whether they heard advice during the alert period (did not hear advice or heard advice); gender; education; household income; region; and location (urban or rural), weighted for non-response using the svy commands.

\section{Focus groups}

Four focus groups, discussing how older people cope in hot weather, were established in three areas in England, chosen to allow for variation in geography and exposure to heat. Participants were purposively selected to include those most at-risk during heatwaves: the majority were female (20 out of 25), all but one were aged $75+$, and most lived alone and had health conditions vulnerable to heat. Participants were recruited through local luncheon clubs and voluntary organisations for older people. We used existing groups on the grounds that this would increase the likelihood of a richer discussion [14].
The topic guide covered attitudes towards hot weather, participants' heat-health behaviours, and any identified risks and coping strategies. A selection of current health promotion leaflets/posters, such as the HWP's 'Beat the Heat' campaign [15], were used as prompts. The topic guide is shown in Additional File 3.

Focus groups were audio-recorded and transcribed verbatim. A thematic analysis was undertaken using the Framework Method [16], whereby themes were identified, both deductively and inductively, from the research questions and participants' narratives. A more detailed explanation of methods is available in [11]. Findings are interwoven with the survey results to provide contextual information for some of the responses in relation to vulnerable adults.

\section{Results}

In total, 1872 respondents were included in the survey analysis, and 95\% $(n=1787)$ reported being in England during the June 2017 level 3 heat-health alert period. Overall, $76 \%$ of the respondents were classified as 'not vulnerable' (group 1), 16\% as 'potentially vulnerable' (group 2) and $8 \%$ as 'vulnerable' (group 3) to hot weather. Table 1 presents respondents' characteristics. This section compares survey results between these three groups, supplemented by findings from the focus groups.

\section{Attitudes to hot weather}

The majority of respondents reported they loved hot weather (57.7\%), while less than a third identified hot weather as a risk to their health (31.1\%) (Table 2). Even among the potentially vulnerable and vulnerable groups, fewer than half identified hot weather as a risk to their health, compared with half saying they loved hot weather.

This attitude to risk was also found in the focus groups, where only a minority of participants said that they felt personally at-risk during heatwaves. In contrast with survey respondents, only a small number of focus group participants voiced positive attitudes towards hot weather, though any negativity expressed largely related to humidity rather than temperature. Participants were, on the whole, nonchalant about heatwaves, viewing them as a rare occurrence in England. "We haven't really had a summer" was a common response.

\section{Views on the effectiveness of protective behaviours}

The proportion of respondents who said the various heat protection measures were very/completely effective was relatively low, although this varied by group (Table 2 ). The not vulnerable and potentially vulnerable groups most frequently reported drinking cool fluids as very/ completely effective (73.6 and $73.3 \%$, respectively). 
Table 1 Overview of survey respondents characteristics (weighted \%)

\begin{tabular}{|c|c|c|c|c|c|}
\hline & $\begin{array}{l}\text { BSA England population } \\
\text { estimate }\end{array}$ & $\begin{array}{l}\text { All } \\
\text { respondents } \\
(n=1872)\end{array}$ & $\begin{array}{l}\text { Not } \\
\text { vulnerable } \\
\text { ( } n=1422)\end{array}$ & $\begin{array}{l}\text { Potentially vulnerable }(n= \\
307)\end{array}$ & $\begin{array}{l}\text { Vulnerable } \\
(n=143)\end{array}$ \\
\hline Age & $\%$ & $\%$ & $\%$ & $\%$ & $\%$ \\
\hline $18-24$ & 11 & 9 & 11 & 7 & - \\
\hline $25-34$ & 17 & 17 & 19 & 15 & - \\
\hline $35-44$ & 17 & 17 & 20 & 15 & - \\
\hline $45-54$ & 18 & 17 & 19 & 17 & - \\
\hline $55-64$ & 14 & 16 & 16 & 26 & - \\
\hline $65+$ & 22 & 28 & 15 & 20 & 100 \\
\hline \multicolumn{6}{|l|}{ Gender } \\
\hline Male & 48 & 48 & 47 & 47 & 56 \\
\hline Female & 52 & 52 & 53 & 53 & 44 \\
\hline \multicolumn{6}{|l|}{ Ethnic group } \\
\hline White & Not available & 87 & 85 & 88 & 98 \\
\hline Other & Not available & 13 & 15 & 12 & 2 \\
\hline \multicolumn{6}{|c|}{ Highest educational attainment } \\
\hline Degree or equivalent & 36 & 33 & 36 & 24 & 22 \\
\hline A level or equivalent & 19 & 22 & 25 & 17 & 14 \\
\hline O level/CSE or equivalent & 26 & 22 & 22 & 28 & 7 \\
\hline Foreign or other & 3 & 8 & 7 & 9 & 18 \\
\hline No qualifications & 17 & 15 & 10 & 23 & 39 \\
\hline
\end{tabular}

Monthly household income

$\begin{array}{ll}\text { Less than } £ 1200 & \text { Not available } \\ £ 1201-£ 2200 & \text { Not available } \\ £ 2201-£ 3700 & \text { Not available } \\ £ 3701 \text { or more } & \text { Not available }\end{array}$

Region

$\begin{array}{ll}\text { North East } & 5 \\ \text { North West } & 13 \\ \text { Yorkshire \& the Humber } & 10 \\ \text { East Midlands } & 9 \\ \text { West Midlands } & 10 \\ \text { East of England } & 11 \\ \text { London } & 16 \\ \text { South East } & 16 \\ \text { South West } & 10\end{array}$

\section{Location}

$\begin{array}{ll}\text { Urban } & \text { Not available } \\ \text { Rural } & \text { Not available }\end{array}$

\section{Household type}

Single person household
Lone parent
2 adults (no children)
2 adults (with children)
$3+$ adults (no children)

$\begin{array}{ll}17 & 17 \\ 4 & 4 \\ 36 & 35 \\ 21 & 23 \\ 15 & 16\end{array}$

$\begin{array}{ll}12 & 26 \\ 4 & 3 \\ 34 & 27 \\ 26 & 20 \\ 17 & 19\end{array}$

37
0
57
0
5


Table 1 Overview of survey respondents characteristics (weighted \%) (Continued)

\begin{tabular}{|c|c|c|c|c|c|}
\hline & $\begin{array}{l}\text { BSA England population } \\
\text { estimate }\end{array}$ & $\begin{array}{l}\text { All } \\
\text { respondents } \\
(n=1872)\end{array}$ & $\begin{array}{l}\text { Not } \\
\text { vulnerable } \\
(n=1422) \\
\end{array}$ & $\begin{array}{l}\text { Potentially vulnerable }(n= \\
307)\end{array}$ & $\begin{array}{l}\text { Vulnerable } \\
(n=143)\end{array}$ \\
\hline $3+$ adults (with children) & 7 & 6 & 7 & 5 & 0 \\
\hline \multicolumn{6}{|l|}{ Social grade } \\
\hline Managerial/professional & 38 & 41 & 43 & 30 & 37 \\
\hline Intermediate & 12 & 14 & 15 & 12 & 11 \\
\hline $\begin{array}{l}\text { Small employers/own account } \\
\text { workers }\end{array}$ & 9 & 8 & 7 & 10 & 8 \\
\hline Lower supervisory/technical & 8 & 8 & 7 & 14 & 10 \\
\hline Semi-routine/routine & 28 & 29 & 27 & 32 & 34 \\
\hline \multicolumn{6}{|l|}{ Health } \\
\hline Good/very good & Not available & 65 & 75 & 24 & 60 \\
\hline Fair & Not available & 26 & 25 & 28 & 32 \\
\hline Bad/very bad & Not available & 9 & 0 & 48 & 8 \\
\hline \multicolumn{6}{|c|}{ Limiting longstanding illness (LLSI) } \\
\hline No & 69 & 67 & 83 & 9 & 45 \\
\hline Does not affect day-to-day life & 15 & 17 & 17 & 8 & 35 \\
\hline Affects day-to-day life & 16 & 16 & 0 & 83 & 20 \\
\hline
\end{tabular}

Among the vulnerable group, staying out of the sun between 11 am and $3 \mathrm{pm}$ was most frequently reported as effective (71.2\%), and was also the behaviour most often mentioned by focus group participants. The behaviour least often considered very/completely effective, across all groups, was closing windows exposed to direct sunlight, ranging from $18.7 \%$ among potentially vulnerable to $27.0 \%$ among the vulnerable. Compared to other groups, the potentially vulnerable were the least likely to rate most behaviours as very/completely effective.

\section{Protective behaviours taken during the level 3 heat- health alert}

While about half of all respondents said they were aware of hot weather-related health publicity or advice during the level 3 heat-health alert period (51.0\%), this increased to about two-thirds in the vulnerable group (63.9\%) (Table 2). However, even among the vulnerable, only $26.8 \%$ reported changing their behaviour as a result of hearing this advice.

Across all three groups, the heat protection measures respondents most frequently reported always/often undertaking were drinking cool fluids (87.3\%) and opening windows at night $(87.1 \%)$ (Table 2). The measures that the fewest respondents reported always/often taking were closing windows exposed to direct sunlight (34.9\%) and using an electric fan (39.3\%). Vulnerable respondents were the most likely to report taking six of the protective behaviours but were less likely to report using an electric fan, opening windows at night and drinking cool fluids. Although we cannot infer cause and effect in our survey, respondents in the vulnerable group were also the least likely to report any adverse health effects from hot weather in 2017: only $31.7 \%$ reported one or more health effects compared to $52.9 \%$ of the not vulnerable group and $62.2 \%$ of the potentially vulnerable group (Table 2).

Results of the multivariable logistic regression analysis focusing on potentially vulnerable and vulnerable groups combined are shown in Table 3. Although the small sample size $(n=431)$ limits the power to detect statistically significant results, there was strong evidence that respondents in these two groups were more likely to undertake a protective behaviour if they believed it to be completely/very effective. The odds ranged from more than twice as great for avoiding the sun (adjusted OR 2.6 [95\% CI 1.5-4.5]) to more than nine times greater for closing exposed windows during the day (adjusted OR 9.6 [95\%CI 4.1-22.5]). Those who reported being in $\mathrm{bad} / \mathrm{very}$ bad health were more likely to avoid the sun (adjusted OR 2.1 [95\%CI 1.0-4.3]), use an electric fan (adjusted OR 3.4 [95\%CI 1.5-7.5]), close exposed windows (adjusted OR 2.2 [95\%CI 1.0-4.8]) and avoid alcohol (adjusted OR 2.8 [95\%CI 1.2-6.3]). Except for avoiding the sun, there was no difference between age groups. Having heard heat protection advice was only associated with closing exposed windows during the day (adjusted OR 1.7 [95\%CI 1.0-3.0]) and opening windows at night (adjusted OR 2.2 [95\%CI 1.1-4.5]), after controlling for other variables.

The focus group findings provide context to explaining behaviours taken, specifically those the survey identified 
Table 2 Attitudes, knowledge, and protective behaviours by type of vulnerable group

\begin{tabular}{lrrrr}
\hline & All & Not vulnerable & Potentially vulnerable & Vulnerable \\
\hline Attitudes to hot weather [\% of respondents that strongly agree or agree $(\mathbf{9 5 \% C l})]$ & & & \\
I love hot weather & $57.7(54.5-60.8)$ & $60.4(56.8-63.8)$ & $49.9(42.0-57.7)$ & $48.7(38.0-59.6)$ \\
Hot weather is a risk to my health & $31.1(28.3-34.1)$ & $26.9(23.9-30.0)$ & $45.3(37.6-53.4)$
\end{tabular}

Effectiveness of heat protection behaviours as [\% of respondents rated behaviour very or completely effective $(95 \% \mathrm{Cl})$ ]

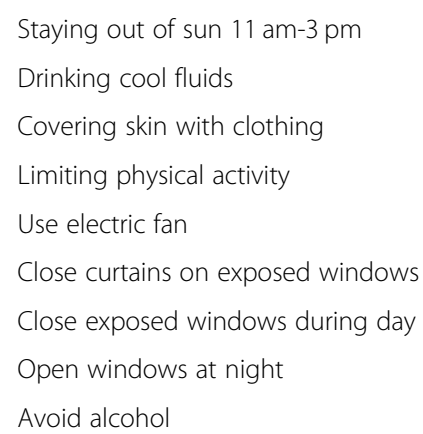

$\begin{array}{lll}66.2(63.0-69.1) & 67.3(63.7-70.6) & 58.8(50.7-66.5) \\ 72.5(69.6-75.3) & 73.6(70.3-76.8) & 73.3(65.6-79.8) \\ 66.4(63.2-69.4) & 65.9(62.2-69.4) & 68.0(59.8-75.2) \\ 64.2(60.9-67.2) & 64.5(60.8-68.0) & 62.3(54.1-69.8) \\ 38.0(34.9-41.2) & 35.4(31.9-39.0) & 51.6(43.8-59.4) \\ 47.6(44.5-50.8) & 47.0(43.4-50.6) & 44.3(36.7-52.2) \\ 20.1(17.6-22.8) & 19.7(16.9-22.8) & 18.7(12.7-26.6) \\ 63.1(60.0-66.1) & 63.9(60.4-67.3) & 58.7(50.6-66.3) \\ 45.2(42.0-48.4) & 45.6(42.0-49.3) & 45.2(37.5-53.2)\end{array}$

$71.2(60.3-80.1)$ $60.8(49.0-71.5)$ $67.6(55.7-77.6)$ $64.8(52.6-75.4)$ $34.0(24.1-45.6)$ $60.5(49.2-70.7)$ $27.0(19.0-36.9)$ $65.1(54.6-74.3)$ $41.2(31.1-52.2)$

Experienced adverse health effects as a result of hot weather or heat [\% of respondents $(95 \% \mathrm{Cl})]$

\begin{tabular}{|c|c|c|c|c|}
\hline None & $47.2(44.1-50.3)$ & $47.1(43.5-50.7)$ & $37.8(30.7-45.4)$ & $68.3(56.7-78.0)$ \\
\hline Dehydration/intense thirst & $20.2(17.6-23.0)$ & $19.8(17.0-23.0)$ & $27.0(19.6-35.8)$ & $8.7(4.8-15.1)$ \\
\hline Sunburn & $18.2(15.6-21.0)$ & $18.7(16.0-21.7)$ & $21.5(14.4-30.8)$ & $6.3(1.3-24.8)$ \\
\hline Heat rash/red and dry skin & $14.0(11.7-16.8)$ & $12.5(10.1-15.3)$ & $22.3(15.4-31.1)$ & $11.1(4.5-24.9)$ \\
\hline Headaches & $25.8(23.0-28.8)$ & $27.1(23.8-30.7)$ & $29.3(22.8-36.8)$ & $5.9(3.0-11.4)$ \\
\hline Dizziness & $9.1(7.3-11.2)$ & $6.8(5.1-9.0)$ & $17.5(12.5-23.9)$ & $12.9(5.6-26.7)$ \\
\hline Nausea or vomiting & $2.9(1.8-4.5)$ & $2.0(1.2-3.3)$ & $5.3(2.2-11.9)$ & $6.1(1.2-25.5)$ \\
\hline Muscle weakness or cramps & $6.4(4.9-8.4)$ & $4.2(3.0-5.8)$ & $16.8(10.9-25.1)$ & $6.2(2.9-12.6)$ \\
\hline A high temperature & $6.5(4.8-8.6)$ & $5.9(4.1-8.4)$ & $8.2(5.2-12.8)$ & $8.3(2.5-24.3)$ \\
\hline Irritability & $21.3(18.7-24.1)$ & $19.2(16.5-22.1)$ & $33.5(25.9-42.0)$ & $15.2(7.6-28.1)$ \\
\hline A need to contact health services (e.g., a GP, an ambulance) & $1.4(0.6-2.9)$ & $0.9(0.3-2.3)$ & $1.0(0.3-4.0)$ & $6.7(1.6-24.4)$ \\
\hline Mean number of adverse health effects & $1.3(0.1)$ & $1.2(0.1)$ & $1.8(1.5)$ & $1.0(0.3)$ \\
\hline Base (unweighted): All respondents & 1872 & 1422 & 307 & 143 \\
\hline \multicolumn{5}{|l|}{ Heard and acted on health advice [\% respondents $(95 \% \mathrm{CI})]$} \\
\hline Not heard advice & $49.0(45.8-52.2)$ & $49.7(46.0-53.5)$ & $51.6(43.6-59.5)$ & $36.1(26.4-47.1)$ \\
\hline Heard advice but did not change behaviour & $29.1(26.3-32.0)$ & $29.7(26.5-33.1)$ & $22.8(17.4-29.4)$ & $37.1(26.3-49.4)$ \\
\hline Heard advice and changed behaviour & $21.9(19.3-24.8)$ & $20.6(17.6-23.9)$ & $25.6(19.2-33.4)$ & $26.8(18.5-37.1)$ \\
\hline \multicolumn{5}{|c|}{ Protective behaviours taken [\% respondents always or often took protective behaviours during 2017 heat alert period $(95 \% \mathrm{Cl})$ ] } \\
\hline Staying out of sun $11 \mathrm{am}-3 \mathrm{pm}$ & $46.6(43.4-49.8)$ & $44.8(41.2-48.5)$ & $48.6(40.7-56.6)$ & $59.2(47.9-68.7)$ \\
\hline Drinking cool fluids & $87.3(84.9-89.4)$ & $88.6(85.9-90.8)$ & $84.8(77.6-90.0)$ & $80.6(71.3-87.4)$ \\
\hline Covering skin with clothing & $50.9(47.7-54.2)$ & $49.3(45.6-53.0)$ & $51.7(43.6-59.6)$ & $65.0(54.2-74.5)$ \\
\hline Limiting physical activity & $56.3(53.1-59.6)$ & $54.5(50.7-58.2)$ & $57.5(49.2-65.4)$ & $71.8(60.4-80.9)$ \\
\hline Use electric fan & $39.3(36.1-42.6)$ & $38.6(35.0-42.3)$ & $46.3(38.4-54.4)$ & $31.0(20.8-43.5)$ \\
\hline Close curtains on exposed windows & $47.7(44.5-51.0)$ & $46.5(42.9-50.3)$ & $49.6(41.6-57.6)$ & $55.3(43.9-66.2)$ \\
\hline Close exposed windows during day & $34.9(31.9-38.0)$ & $33.2(29.9-36.7)$ & $34.3(26.8-42.8)$ & $52.4(41.3-63.3)$ \\
\hline Open windows at night & $87.1(84.5-89.3)$ & $88.2(85.1-90.8)$ & $86.2(80.3-90.5)$ & $78.4(69.1-85.5)$ \\
\hline Avoid alcohol & $51.2(47.9-54.4)$ & $47.7(44.0-51.5)$ & $61.1(52.6-68.9)$ & $62.8(52.2-72.3)$ \\
\hline Mean number of protective behaviours always/often taken (sd) & $5.0(2.1)$ & $4.9(2.1)$ & $5.2(2.0)$ & $5.6(2.2)$ \\
\hline Base (unweighted): In England during level 3 heat alert & 1787 & 1356 & 295 & 136 \\
\hline
\end{tabular}


Table 3 Multivariable regression analysis examining heat protection behaviours always/often taken by potentially vulnerable and vulnerable groups $(n=431)$

\begin{tabular}{|c|c|c|c|c|c|c|c|c|c|}
\hline & $\begin{array}{l}\text { Avoid } \\
\text { sun } \\
\text { OR } \\
(95 \% \mathrm{Cl})\end{array}$ & $\begin{array}{l}\text { Drink } \\
\text { cool } \\
\text { fluids } \\
\text { OR } \\
(95 \% \mathrm{Cl})\end{array}$ & $\begin{array}{l}\text { Covers } \\
\text { skin } \\
\text { OR } \\
(95 \% \mathrm{Cl})\end{array}$ & $\begin{array}{l}\text { Limits } \\
\text { physical } \\
\text { activity } \\
\text { OR }(95 \% \mathrm{Cl})\end{array}$ & $\begin{array}{l}\text { Electric } \\
\text { fan } \\
\text { OR } \\
(95 \% \mathrm{Cl})\end{array}$ & $\begin{array}{l}\text { Close curtain on } \\
\text { exposed windows day } \\
\text { OR }(95 \% \mathrm{Cl})\end{array}$ & $\begin{array}{l}\text { Close exposed } \\
\text { windows day } \\
\text { OR }(95 \% \mathrm{Cl})\end{array}$ & $\begin{array}{l}\text { Open } \\
\text { windows } \\
\text { night } \\
\text { OR }(95 \% \mathrm{Cl})\end{array}$ & $\begin{array}{l}\text { Avoid } \\
\text { alcohol } \\
\text { OR } \\
(95 \% \mathrm{Cl})\end{array}$ \\
\hline \multicolumn{10}{|l|}{$\overline{\text { Age }}$} \\
\hline $18-34$ & 1 & 1 & 1 & 1 & 1 & 1 & 1 & 1 & 1 \\
\hline $35-54$ & $\begin{array}{l}1.7(0.7- \\
4.2)\end{array}$ & $\begin{array}{l}0.4(0.0- \\
5.5)\end{array}$ & $\begin{array}{l}0.4(0.1- \\
1.1)\end{array}$ & $0.5(0.2-1.6)$ & $\begin{array}{l}0.2 \\
(0.1- \\
0.7)\end{array}$ & $2.2(0.8-6.2)$ & $0.7(0.3-1.9)$ & $1.6(0.4-6.2)$ & $\begin{array}{l}0.9(0.3- \\
2.8)\end{array}$ \\
\hline $55-74$ & $\begin{array}{l}2.5 \\
(1.0- \\
6.5)\end{array}$ & $\begin{array}{l}0.4(0.0- \\
5.8)\end{array}$ & $\begin{array}{l}1.0(0.3- \\
2.7)\end{array}$ & $0.6(0.2-1.9)$ & $\begin{array}{l}0.3 \\
(0.1- \\
0.9)\end{array}$ & $3.2(1.1-9.1)$ & $1.3(0.5-3.6)$ & $0.8(0.2-2.9)$ & $\begin{array}{l}1.0(0.3- \\
3.0)\end{array}$ \\
\hline 75 and over & $\begin{array}{l}4.1 \\
(1.2- \\
14.7)\end{array}$ & $\begin{array}{l}0.3(0.0- \\
4.6)\end{array}$ & $\begin{array}{l}0.8(0.2- \\
2.6)\end{array}$ & $2.2(0.6-8.0)$ & $\begin{array}{l}0.4(0.1- \\
1.8)\end{array}$ & $1.7(0.5-5.9)$ & $1.4(0.4-4.6)$ & $0.3(0.1-1.2)$ & $\begin{array}{l}2.0(0.5- \\
7.5)\end{array}$ \\
\hline \multicolumn{10}{|l|}{ Health } \\
\hline Good/very good & 1 & 1 & 1 & 1 & 1 & 1 & 1 & 1 & 1 \\
\hline Fair & $\begin{array}{l}1.8(0.8- \\
3.8)\end{array}$ & $\begin{array}{l}0.8(0.3- \\
2.3)\end{array}$ & $\begin{array}{l}1.2(0.6- \\
2.4)\end{array}$ & $1.3(0.6-2.8)$ & $\begin{array}{l}3.0 \\
(1.3- \\
6.8)\end{array}$ & $1.4(0.7-2.9)$ & $2.1(1.0-4.5)$ & $1.3(0.5-3.3)$ & $\begin{array}{l}2.3 \\
(1.1- \\
5.2)\end{array}$ \\
\hline Bad/very bad & $\begin{array}{l}2.1 \\
(1.0- \\
4.3)\end{array}$ & $\begin{array}{l}0.6(0.2- \\
1.4)\end{array}$ & $\begin{array}{l}0.8(0.4- \\
1.7)\end{array}$ & $1.4(0.6-3.0)$ & $\begin{array}{l}3.4 \\
(1.5- \\
7.5)\end{array}$ & $1.5(0.7-3.2)$ & $2.2(1.0-4.8)$ & $0.8(0.3-1.9)$ & $\begin{array}{l}2.8 \\
(1.2- \\
6.3)\end{array}$ \\
\hline \multicolumn{10}{|l|}{ LLSI } \\
\hline No & 1 & 1 & 1 & 1 & 1 & 1 & 1 & 1 & 1 \\
\hline $\begin{array}{l}\text { Does not affect } \\
\text { day-to-day life }\end{array}$ & $\begin{array}{l}0.4 \\
(0.1- \\
1.0)\end{array}$ & $\begin{array}{l}1.2(0.4- \\
3.6)\end{array}$ & $\begin{array}{l}0.7(0.3- \\
2.0)\end{array}$ & $0.7(0.3-1.7)$ & $\begin{array}{l}0.5(0.2- \\
1.5)\end{array}$ & $0.8(0.3-2.0)$ & $0.8(0.3-2.1)$ & $0.8(0.2-2.4)$ & $\begin{array}{l}1.2(0.5- \\
3.0)\end{array}$ \\
\hline $\begin{array}{l}\text { Affects day-to- } \\
\text { day life }\end{array}$ & $\begin{array}{l}0.8(0.3- \\
1.9)\end{array}$ & $\begin{array}{l}0.9(0.4- \\
2.5)\end{array}$ & $\begin{array}{l}0.4(0.2- \\
1.0)\end{array}$ & $0.9(0.4-2.1)$ & $\begin{array}{l}0.6(0.3- \\
1.6)\end{array}$ & $0.7(0.3-1.7)$ & $0.3(0.1-0.7)$ & $0.5(0.2-1.4)$ & $\begin{array}{l}1.5(0.6- \\
3.7)\end{array}$ \\
\hline \multicolumn{10}{|l|}{ Household type } \\
\hline $\begin{array}{l}\text { Living with } \\
\text { someone }\end{array}$ & 1 & 1 & 1 & 1 & 1 & 1 & 1 & 1 & 1 \\
\hline Living alone & $\begin{array}{l}0.9(0.5- \\
1.7)\end{array}$ & $\begin{array}{l}1.0(0.5- \\
2.3)\end{array}$ & $\begin{array}{l}0.9(0.5- \\
1.7)\end{array}$ & $0.7(0.4-1.4)$ & $\begin{array}{l}0.4 \\
(0.2- \\
0.8)\end{array}$ & $1.1(0.6-2.2)$ & $1.2(0.7-2.4)$ & $0.5(0.2-1.0)$ & $\begin{array}{l}0.4 \\
(0.2- \\
0.8)\end{array}$ \\
\hline \multicolumn{10}{|l|}{ Heard alert advice } \\
\hline $\begin{array}{l}\text { Did not hear } \\
\text { advice }\end{array}$ & 1 & 1 & 1 & 1 & 1 & 1 & 1 & 1 & 1 \\
\hline Heard advice & $\begin{array}{l}1.4(0.8- \\
2.4)\end{array}$ & $\begin{array}{l}1.9(0.9- \\
3.7)\end{array}$ & $\begin{array}{l}1.1(0.6- \\
1.9)\end{array}$ & $1.0(0.6-1.9)$ & $\begin{array}{l}1.3(0.7- \\
2.5)\end{array}$ & $1.0(0.6-1.8)$ & $1.7(1.0-3.0)$ & $2.2(1.1-4.5)$ & $\begin{array}{l}1.4(0.8- \\
2.4)\end{array}$ \\
\hline \multicolumn{10}{|l|}{ Effective } \\
\hline $\begin{array}{l}\text { Not/slightly/ } \\
\text { somewhat }\end{array}$ & 1 & 1 & 1 & 1 & 1 & 1 & 1 & 1 & 1 \\
\hline Completely/very & $\begin{array}{l}2.6 \\
(1.5- \\
4.5)\end{array}$ & $\begin{array}{l}6.2(2.8- \\
13.7)\end{array}$ & $\begin{array}{l}2.8 \\
(1.5- \\
5.1)\end{array}$ & $3.7(2.0-7.0)$ & $\begin{array}{l}4.9 \\
(2.7- \\
9.1)\end{array}$ & $6.5(3.7-11.4)$ & $9.6(4.1-22.5)$ & $3.7(1.6-8.4)$ & $\begin{array}{l}4.3 \\
(2.4- \\
7.8)\end{array}$ \\
\hline \multicolumn{10}{|c|}{ Hot weather is a risk to health } \\
\hline $\begin{array}{l}\text { Disagree/ } \\
\text { strongly } \\
\text { disagree }\end{array}$ & 1 & 1 & 1 & 1 & 1 & 1 & 1 & 1 & 1 \\
\hline Neutral & $\begin{array}{l}1.3(0.6- \\
2.6)\end{array}$ & $\begin{array}{l}1.6(0.6- \\
4.7)\end{array}$ & $\begin{array}{l}1.0(0.5- \\
1.9)\end{array}$ & $1.0(0.4-2.2)$ & $\begin{array}{l}1.4(0.6- \\
3.2)\end{array}$ & $2.2(1.0-4.7)$ & $2.5(1.2-5.3)$ & $0.5(0.2-1.2)$ & $\begin{array}{l}1.4(0.7- \\
2.8)\end{array}$ \\
\hline
\end{tabular}


Table 3 Multivariable regression analysis examining heat protection behaviours always/often taken by potentially vulnerable and vulnerable groups $(n=431)$ (Continued)

\begin{tabular}{|c|c|c|c|c|c|c|c|c|c|}
\hline & $\begin{array}{l}\text { Avoid } \\
\text { sun } \\
\text { OR } \\
(95 \% \mathrm{Cl})\end{array}$ & $\begin{array}{l}\text { Drink } \\
\text { cool } \\
\text { fluids } \\
\text { OR } \\
(95 \% \mathrm{Cl})\end{array}$ & $\begin{array}{l}\text { Covers } \\
\text { skin } \\
\text { OR } \\
(95 \% \mathrm{Cl})\end{array}$ & $\begin{array}{l}\text { Limits } \\
\text { physical } \\
\text { activity } \\
\text { OR }(95 \% \mathrm{Cl})\end{array}$ & $\begin{array}{l}\text { Electric } \\
\text { fan } \\
\text { OR } \\
(95 \% \mathrm{Cl})\end{array}$ & $\begin{array}{l}\text { Close curtain on } \\
\text { exposed windows day } \\
\text { OR }(95 \% \mathrm{Cl})\end{array}$ & $\begin{array}{l}\text { Close exposed } \\
\text { windows day } \\
\text { OR }(95 \% \mathrm{Cl})\end{array}$ & $\begin{array}{l}\text { Open } \\
\text { windows } \\
\text { night } \\
\text { OR }(95 \% \mathrm{Cl})\end{array}$ & $\begin{array}{l}\text { Avoid } \\
\text { alcohol } \\
\text { OR } \\
(95 \% \mathrm{Cl})\end{array}$ \\
\hline $\begin{array}{l}\text { Agree/strongly } \\
\text { agree }\end{array}$ & $\begin{array}{l}1.9(1.0- \\
3.7)\end{array}$ & $\begin{array}{l}1.4(0.5- \\
3.9)\end{array}$ & $\begin{array}{l}1.3(0.6- \\
2.7)\end{array}$ & $2.1(1.0-4.3)$ & $\begin{array}{l}1.3(0.6- \\
2.7)\end{array}$ & $1.3(0.6-2.7)$ & $1.4(0.6-3.0)$ & $0.7(0.3-1.6)$ & $\begin{array}{l}1.0(0.5- \\
2.0)\end{array}$ \\
\hline \multicolumn{10}{|l|}{ Love hot weather } \\
\hline $\begin{array}{l}\text { Disagree/ } \\
\text { strongly } \\
\text { disagree }\end{array}$ & 1 & 1 & 1 & 1 & 1 & 1 & 1 & 1 & 1 \\
\hline Neutral & $\begin{array}{l}0.8(0.4- \\
1.8)\end{array}$ & $\begin{array}{l}1.3(0.4- \\
4.2)\end{array}$ & $\begin{array}{l}1.0(0.4- \\
2.3)\end{array}$ & $0.5(0.2-1.1)$ & $\begin{array}{l}0.4 \\
(0.2- \\
1.0)\end{array}$ & $0.4(0.1-0.9)$ & $1.2(0.5-2.9)$ & $0.4(0.1-1.0)$ & $\begin{array}{l}0.9(0.4- \\
2.0)\end{array}$ \\
\hline $\begin{array}{l}\text { Agree/strongly } \\
\text { agree }\end{array}$ & $\begin{array}{l}0.8(0.3- \\
1.6)\end{array}$ & $\begin{array}{l}1.2(0.4- \\
3.6)\end{array}$ & $\begin{array}{l}0.4 \\
(0.2- \\
0.9)\end{array}$ & $0.6(0.3-1.3)$ & $\begin{array}{l}0.9(0.4- \\
1.9)\end{array}$ & $0.4(0.2-0.8)$ & $1.1(0.5-2.5)$ & $0.6(0.2-1.7)$ & $\begin{array}{l}0.5(0.2- \\
1.2)\end{array}$ \\
\hline \multicolumn{10}{|l|}{ Sex } \\
\hline Male & 1 & 1 & 1 & 1 & 1 & 1 & 1 & 1 & 1 \\
\hline Female & $\begin{array}{l}1.9 \\
(1.1- \\
3.3)\end{array}$ & $\begin{array}{l}1.8(0.9- \\
3.8)\end{array}$ & $\begin{array}{l}0.8(0.5- \\
1.5)\end{array}$ & $1.8(0.9-3.4)$ & $\begin{array}{l}1.1(0.6- \\
2.0)\end{array}$ & $1.3(0.7-2.3)$ & $0.9(0.5-1.6)$ & $1.2(0.5-2.6)$ & $\begin{array}{l}1.9 \\
(1.1- \\
3.3)\end{array}$ \\
\hline \multicolumn{10}{|l|}{ Ethnicity } \\
\hline White & 1 & 1 & 1 & 1 & 1 & 1 & 1 & 1 & 1 \\
\hline Other & $\begin{array}{l}2.1(0.7- \\
6.6)\end{array}$ & $\begin{array}{l}0.4(0.1- \\
1.5)\end{array}$ & $\begin{array}{l}0.3(0.1- \\
1.1)\end{array}$ & $1.7(0.6-5.1)$ & $\begin{array}{l}1.1(0.3- \\
3.9)\end{array}$ & $1.3(0.3-5.1)$ & $1.2(0.4-4.1)$ & $0.7(0.1-3.7)$ & $\begin{array}{l}4.4(1.1- \\
17.2)\end{array}$ \\
\hline \multicolumn{10}{|l|}{ Education } \\
\hline Degree/higher & 1 & 1 & 1 & 1 & 1 & 1 & 1 & 1 & 1 \\
\hline $\begin{array}{l}\text { A-level/ } \\
\text { equivalent }\end{array}$ & $\begin{array}{l}0.4(0.2- \\
1.1)\end{array}$ & $\begin{array}{l}1.6(0.5- \\
4.8)\end{array}$ & $\begin{array}{l}0.6(0.3- \\
1.3)\end{array}$ & $0.3(0.1-0.7)$ & $\begin{array}{l}1.9(0.7- \\
4.8)\end{array}$ & $0.7(0.3-1.6)$ & $0.7(0.3-1.8)$ & $1.7(0.3-8.0)$ & $\begin{array}{l}0.5(0.2- \\
1.2)\end{array}$ \\
\hline $\begin{array}{l}\text { Below A-level/ } \\
\text { none }\end{array}$ & $\begin{array}{l}0.5(0.2- \\
1.1)\end{array}$ & $\begin{array}{l}1.4(0.6- \\
3.4)\end{array}$ & $\begin{array}{l}0.7(0.3- \\
1.5)\end{array}$ & $0.6(0.2-1.3)$ & $\begin{array}{l}1.9(0.7- \\
3.9)\end{array}$ & $0.5(0.2-1.1)$ & $1.0(0.4-2.3)$ & $0.5(0.2-1.5)$ & $\begin{array}{l}1.1(0.5- \\
2.3)^{(1)}\end{array}$ \\
\hline Other & $\begin{array}{l}0.8(0.3- \\
2.3)\end{array}$ & $\begin{array}{l}4.9(1.0- \\
23.7)\end{array}$ & $\begin{array}{l}1.2(0.4- \\
3.9)\end{array}$ & $0.7(0.2-2.3)$ & $\begin{array}{l}2.5(1.0- \\
6.5)\end{array}$ & $0.9(0.4-2.4)$ & $1.5(0.4-4.9)$ & $1.5(0.3-7.5)$ & $\begin{array}{l}1.2(0.4- \\
3.6)\end{array}$ \\
\hline \multicolumn{10}{|c|}{ Monthly household income } \\
\hline Less than $£ 1200$ & 1 & 1 & 1 & 1 & 1 & 1 & 1 & 1 & 1 \\
\hline$£ 1201-£ 2200$ & $\begin{array}{l}1.1(0.6- \\
2.3)^{(1)}\end{array}$ & $\begin{array}{l}0.8(0.3- \\
2.0)\end{array}$ & $\begin{array}{l}0.9(0.5- \\
1.8)\end{array}$ & $1.3(0.6-2.9)$ & $\begin{array}{l}0.6(0.3- \\
1.3)\end{array}$ & $1.0(0.5-2.1)$ & $0.9(0.5-1.9)$ & $0.7(0.3-1.7)$ & $\begin{array}{l}1.7(0.8- \\
3.7)\end{array}$ \\
\hline$£ 2201-£ 3700$ & $\begin{array}{l}1.5(0.7- \\
3.4)\end{array}$ & $\begin{array}{l}1.8(0.5- \\
6.2)\end{array}$ & $\begin{array}{l}1.7(0.8- \\
3.6)\end{array}$ & $1.9(0.9-4.2)$ & $\begin{array}{l}1.2(0.5- \\
2.8)\end{array}$ & $0.8(0.4-1.9)$ & $0.6(0.2-1.3)$ & $1.3(0.5-3.5)$ & $\begin{array}{l}0.7(0.3- \\
1.7)\end{array}$ \\
\hline$£ 3701$ or more & $\begin{array}{l}0.4(0.1- \\
1.1)\end{array}$ & $\begin{array}{l}5.0(1.1- \\
22.1)\end{array}$ & $\begin{array}{l}2.2(0.8- \\
6.0)\end{array}$ & $3.0(1.0-9.2)$ & $\begin{array}{l}2.8 \\
(1.1- \\
7.2)\end{array}$ & $0.6(0.2-1.6)$ & $1.4(0.5-3.9)$ & $\begin{array}{l}6.8(1.0- \\
47.0)\end{array}$ & $\begin{array}{l}0.4(0.2- \\
1.2)\end{array}$ \\
\hline \multicolumn{10}{|l|}{ Region } \\
\hline North & 1 & 1 & 1 & 1 & 1 & 1 & 1 & 1 & 1 \\
\hline Midlands & $\begin{array}{l}1.3(0.6- \\
2.6)\end{array}$ & $\begin{array}{l}1.9(0.5- \\
7.4)\end{array}$ & $\begin{array}{l}0.8(0.4- \\
1.8)\end{array}$ & $1.1(0.5-2.5)$ & $\begin{array}{l}2.2 \\
(1.0- \\
4.9)\end{array}$ & $1.5(0.7-3.4)$ & $0.6(0.3-1.3)$ & $0.8(0.3-2.0)$ & $\begin{array}{l}1.1(0.5- \\
2.4)\end{array}$ \\
\hline East & $\begin{array}{l}0.8(0.3- \\
1.9)\end{array}$ & $\begin{array}{l}0.6(0.2- \\
1.7)\end{array}$ & $\begin{array}{l}0.6(0.3- \\
1.6)\end{array}$ & $1.5(0.6-4.1)$ & $\begin{array}{l}1.9(0.7- \\
5.2)\end{array}$ & $1.4(0.6-3.4)$ & $0.6(0.2-1.7)$ & $0.7(0.2-2.1)$ & $\begin{array}{l}1.3(0.5- \\
3.1)\end{array}$ \\
\hline $\begin{array}{l}\text { London \& South } \\
\text { East }\end{array}$ & $\begin{array}{l}0.9(0.4- \\
2.0)\end{array}$ & $\begin{array}{l}1.4(0.5- \\
3.8)\end{array}$ & $\begin{array}{l}0.7(0.3- \\
1.4)\end{array}$ & $0.9(0.4-2.1)$ & $\begin{array}{l}2.1(1.0- \\
4.4)\end{array}$ & $2.4(1.1-5.3)$ & $0.7(0.3-1.5)$ & $0.8(0.3-2.2)$ & $\begin{array}{l}0.8(0.4- \\
1.6)\end{array}$ \\
\hline South West & $\begin{array}{l}1.8(0.6- \\
4.8)\end{array}$ & $\begin{array}{l}1.0(0.3- \\
3.7)\end{array}$ & $\begin{array}{l}0.7(0.3- \\
2.0)\end{array}$ & $1.0(0.4-2.7)$ & $\begin{array}{l}1.0(0.3- \\
3.5)\end{array}$ & $1.2(0.5-3.1)$ & $0.4(0.1-1.3)$ & $\begin{array}{l}22.6(2.1- \\
248.1)\end{array}$ & $\begin{array}{l}2.5(0.8- \\
8.1)\end{array}$ \\
\hline
\end{tabular}


Table 3 Multivariable regression analysis examining heat protection behaviours always/often taken by potentially vulnerable and vulnerable groups $(n=431)$ (Continued)

\begin{tabular}{|c|c|c|c|c|c|c|c|c|c|}
\hline & $\begin{array}{l}\text { Avoid } \\
\text { sun } \\
\text { OR } \\
(95 \% \mathrm{Cl})\end{array}$ & $\begin{array}{l}\text { Drink } \\
\text { cool } \\
\text { fluids } \\
\text { OR } \\
(95 \% \mathrm{Cl})\end{array}$ & $\begin{array}{l}\text { Covers } \\
\text { skin } \\
\text { OR } \\
(95 \% \mathrm{Cl})\end{array}$ & $\begin{array}{l}\text { Limits } \\
\text { physical } \\
\text { activity } \\
\text { OR }(95 \% \mathrm{Cl})\end{array}$ & $\begin{array}{l}\text { Electric } \\
\text { fan } \\
\text { OR } \\
(95 \% \mathrm{Cl})\end{array}$ & $\begin{array}{l}\text { Close curtain on } \\
\text { exposed windows day } \\
\text { OR }(95 \% \mathrm{Cl})\end{array}$ & $\begin{array}{l}\text { Close exposed } \\
\text { windows day } \\
\text { OR }(95 \% \mathrm{Cl})\end{array}$ & $\begin{array}{l}\text { Open } \\
\text { windows } \\
\text { night } \\
\text { OR }(95 \% \mathrm{Cl})\end{array}$ & $\begin{array}{l}\text { Avoid } \\
\text { alcohol } \\
\text { OR } \\
(95 \% \mathrm{Cl})\end{array}$ \\
\hline \multicolumn{10}{|l|}{ Location } \\
\hline Rural & 1 & 1 & 1 & 1 & 1 & 1 & 1 & 1 & 1 \\
\hline Urban & $\begin{array}{l}0.9(0.4- \\
1.7)\end{array}$ & $\begin{array}{l}1.5(0.7- \\
3.3)\end{array}$ & $\begin{array}{l}0.9(0.4- \\
1.9)\end{array}$ & $1.0(0.5-1.9)$ & $\begin{array}{l}1.4(0.6- \\
3.5)\end{array}$ & $0.8(0.4-1.7)$ & $1.9(0.9-3.9)$ & $1.3(0.5-3.0)$ & $\begin{array}{l}1.5(0.7- \\
3.1)\end{array}$ \\
\hline
\end{tabular}

Note: Each logistic regression model controls for all covariates shown in the table Bold text $p<0.05$

as being less likely to be taken by vulnerable groups. Participants believed they had good knowledge of actions to take in a heatwave; it was, for them, "common sense", and few felt they belonged to any special risk category. Most said they stayed out of the sun and covered their skin. For many this was to avoid harmful effects of ultraviolet rays, as, according to some participants, ageing "thinned the skin", thereby making it more susceptible to sun damage. Other measures, such as opening windows at night, drinking extra fluids, or using a fan, were inconsistently applied or absent. Risk awareness, security, and costs were identified as key reasons.

A strong theme was safety and security. Most participants kept their windows closed during hot nights for fear of intruders, opting to endure any short-lived discomfort and appearing unaware of any added risks to their health. Cost and noise were identified as reasons for not employing electric fans, particularly at night. Others spoke of their lack of knowledge of some protective measures, such as closing and shading sun-facing windows during the day, or not being aware of raised indoor temperatures, expressing surprise when visitors told them "it's really hot in here". Additionally, even though all agreed that maintaining hydration was important in hot weather, some admitted that they did not drink enough, yet were unconcerned about it as they "did not often feel thirsty".

\section{Discussion}

\section{Main findings}

Heat protection messages often target older people and those in poor health because of their greater vulnerability to heat-related harm [17]. Our results show that many vulnerable and potentially vulnerable adults do not consider hot weather to be a risk to their health. They also illustrate that a person's attitude to hot weather appears to shape their behaviour, including those in vulnerable and potentially vulnerable groups, which accords with results from previous studies among the general population [18-21]. Moreover, much current public health messaging does not appear to be having the desired effect. Only around a quarter of respondents in the potentially vulnerable and vulnerable groups reported changing their behaviour during the level 3 heat-health alert period in June 2017 as a result of having heard hot weather-related health advice.

The focus groups help interpret these survey findings. Individual narratives of personal health risks during heatwaves frequently referred to the effects of the sun's ultra-violet radiation on the skin, rather than to the effects of hot temperatures on health. This has been noted in a previous study [18] and is reflected in current guidance provided by a national charity advising older people [22], which gives primacy to information about sun and skin health. As long as they stayed in the house, or in the shade, participants did not identify themselves as 'atrisk', and were less likely to take other actions to protect themselves from heat. This also explains why some felt it was inappropriate for them to be identified as 'vulnerable' by health and social care services, as in the HWP. These findings support those found in another recent study [23] showing older people being less likely than younger people to be aware of the risks of hot weather and taking appropriate actions.

For four of the nine heat protection behaviours examined, a majority of the potentially vulnerable and vulnerable groups were not aware of their effectiveness, including closing exposed windows during the day, closing curtains on exposed windows during the day (potentially vulnerable group only), using an electric fan and avoiding alcohol. Raising awareness is important as our results show that even people in the potentially vulnerable and vulnerable groups who recognised the risk of hot weather were much less likely to take protective measures if they did not consider the behaviours to be effective. This lack of awareness means many potentially vulnerable adults are not taking all the measures they should to protect themselves from the potentially harmful health effects of hot weather. 


\section{Limitations of this study}

For the survey analysis, apart from people aged $75+$, we could only approximate which individuals may be vulnerable to adverse health impacts of hot weather. We used the term 'potentially vulnerable' to indicate that some members included in this group may not actually be at-risk since we do not know, for example, whether those reporting a LLSI had a chronic condition that increased their health risk during hot weather.

The study findings on behaviours taken and adverse health effects are based on self-reports and thus may be subject to mis-reports and recall bias, especially as the survey and focus groups took place several months after the June 2017 heat-health alert period. Another limitation is that the survey response rate was $60 \%$, and it is possible that vulnerable adults in poorer health may have been less likely to complete the questionnaire, although corrective weighting was undertaken to limit the effects of non-response bias.

To limit sensitivity bias, focus group participants were instructed that they should only reference personal information that they felt happy to share within the group and were not prompted to do so. This may have led to some under-reporting of health or social conditions that might have influenced responses. However, as all groups were 'natural' groups, in that they met regularly through their luncheon or tea groups, participants appeared to speak freely about their health and social conditions during the interviews. As a result of the focus groups being 'natural' groups, it was not possible to balance the groups in terms of gender, and there were more female participants as a result.

\section{Conclusions}

Previous research has shown that the majority of the population does not change behaviour as a result of receiving advice during heat-health alert periods. Those who have positive views about hot weather are less likely to perceive themselves to be at risk of hot weather and to take protective actions; and individuals are more likely to take actions they perceive to be effective. Our study demonstrates that these findings also apply to groups generally considered vulnerable or potentially vulnerable to the effects of hot weather, including people aged $75+$. In particular, these individuals do not see themselves as at-risk in hot weather and are unaware of the effectiveness of a number of important heat protective behaviours. Even vulnerable individuals who are aware of the dangers of hot weather to their health are unlikely to take protective actions if they do not recognise their effectiveness. Moreover, public health messages should avoid labelling individuals as 'vulnerable' [20], when raising awareness of the risks of hot weather even among those who fit the definition. Also, since high proportions of potentially vulnerable members of the population are not aware of the effectiveness of a number of important heat protection measures, there remains considerable scope to increase the uptake of these measures.

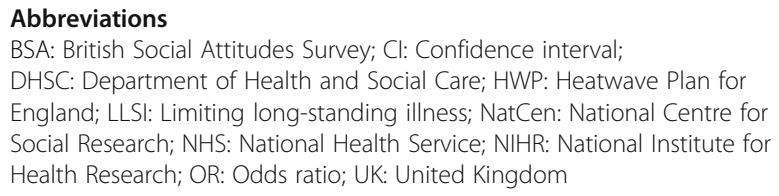

\section{A. Additional files}

\section{Acknowledgements \\ The authors would like to thank all those members of the public who gave up their time to participate in the survey and focus groups. We are also grateful to our lay advisors who provided feedback on draft documents; NatCen colleagues, especially Curtis Jessop, for their help with the survey; and the charitable organisations who helped set up the focus groups, in particular Sharon Frankland and colleagues from Contact the Elderly.}

\section{Authors' contributions}

BE, LW, SE, TM, SH and NM: study conceptualisation and design. BE, LW and JE: data analysis and manuscript drafting. SE, TM, SH and NM: reviewed and revised manuscript. All authors have read and approved the final manuscript.

\section{Funding}

This work was supported by the National Institute for Health Research (NIHR) Policy Research Programme, conducted through the Policy Research Unit in Policy Innovation and Evaluation [reference 102/0001]. The funder had no role in the design of the study, in the collection, analysis and interpretation of the data, or in writing this article. The views expressed are those of the author(s) and not necessarily those of the NIHR or the Department of Health and Social Care.

\section{Availability of data and materials}

The data used and/or analysed for this article are available from the corresponding author on reasonable request.

\section{Declarations}

\section{Ethics approval and consent to participate}

The study received ethical approval from the London School of Hygiene \& Tropical Medicine's Research Ethics Committee on 21st March 2017 (reference 1204-1). Since all survey participants were signed-up members of a long-standing survey panel, the ethics committee approved a process which would evidence respondent consent by their voluntary act of completing the online questionnaire or by answering the questions through a telephone interview. All focus group participants provided signed consent.

\section{Consent for publication}

Not applicable.

\section{Competing interests}

The authors declare that they have no competing interests.

\section{Author details}

${ }^{1}$ Policy Innovation \& Evaluation Research Unit, Department of Health Services Research \& Policy, London School of Hygiene \& Tropical Medicine, London WC1H 9SH, UK. ²Department of Public Health, Environments \& Society, London School of Hygiene \& Tropical Medicine, London WC1H 9SH, UK.

Received: 7 May 2021 Accepted: 26 August 2021

Published online: 06 September 2021

\section{References}

1. Arbuthnott KG, Hajat S. The health effects of hotter summers and heat waves in the population of the United Kingdom: a review of the evidence. 
Environ Health. 2017;16(Suppl 1):119. https://doi.org/10.1186/s12940-0170322-5.

2. Kovats RS, Johnson H, Griffith C. Mortality in southern England during the 2003 Heat wave by place of death. Health Stat Q. 2006;29:6-8.

3. Hajat S, Vardoulakis $\mathrm{S}$, Heaviside C, Eggen B. Climate change effects on human health: projections of temperature-related mortality for the UK during the 2020s, 2050s and 2080s. J Epidemiol Community Health. 2014; 68(7):641-8. https://doi.org/10.1136/jech-2013-202449.

4. Public Health England. The Heatwave Plan for England, Public Health England, Department of Health and social Care, NHS England London; 2018.

5. Heatwave Plan for England. Public Health England, Department of Health, NHS England [press release]. May 20152015.

6. Howarth C, Kantenbacher J, Guida K, Roberts T, Rohse M. Improving resilience to hot weather in the UK: the role of communication, behaviour and social insights in policy interventions. Environ Sci Pol. 2019;94:258-61. https://doi.org/10.1016/j.envsci.2019.01.008.

7. Kenney WL, Hodgson JL. Heat tolerance, Thermoregulation and Ageing. Sports Med. 1987;4(6):446-56. https://doi.org/10.2165/00007256-19870406000004.

8. Public Health England. Heatwave 2013: Evaluation of health impacts and responses. Unpublished; 2016.

9. Khare $S$, Hajat $S$, Kovats $S$, Lefevre $C E$, de Bruin WB, Dessai $S$, et al. Heat protection behaviour in the UK: results of an online survey after the 2013 heatwave. BMC Public Health. 2015;15(1):878. https://doi.org/10.1186/s12 889-015-2181-8.

10. Lefevre CE, Bruine de Bruin W, Taylor AL, Dessai S, Kovats S, Fischhoff B. Heat protection behaviors and positive affect about heat during the 2013 heat wave in the United Kingdom. Soc Sci Med. 2015;128:282-9.

11. Williams L, Erens B, Ettelt S, Hajat S, Mannacorda T, Mays N. Evaluation of the Heatave plan for England: final report. London: London School of Hygiene and Tropical Medicine; 2019. https://piru.ac.uk/assets/files/Evalua tion\%20of\%20the\%20Heatwave\%20Plan\%20for\%20England\%20-\%20Final\%2 OReport.pdf

12. Public Health England. PHE Heatwave mortality monitoring: Summer 2017 London2018 [updated January 2019. Available from: https://assets. publishing.service.gov.uk/government/uploads/system/uploads/attachment_ data/file/771810/PHE_heatwave_mortality_monitoring_report_2017.pdf.

13. Jessop C. The NatCen Panel: developing an open probability-based mixed modes panel in Great Britain. Social Research Practice (6). http://the-sra.org uk/journal-social-research-practice/ 2018 [.

14. Kitzinger J. The methodology of focus groups: the importance of interaction between research participants. Sociol Health IIIness. 1994;16(1):103-21. https://doi.org/10.1111/1467-9566.ep11347023.

15. Public Health England. Beat the Heat: staying safe in hot weather 2017 [cited 2017. Available from: https://assets.publishing.service.gov.uk/ government/uploads/system/uploads/attachment_data/file/731044/2018_ Beat_the_Heat_Leaflet.pdf.

16. Ritchie J, Spencer L. Qualitative data analysis for applied policy research. In: ABaRG B, editor. Analysing qualitative data. London: Routledge; 1994. p. 173-94.

17. Kovats RS, Hajat S. Heat stress and public health: a critical review. Annu Rev Public Health. 2008;29(1):41-55. https://doi.org/10.1146/annurev.publhealth.2 9.020907.090843

18. Wolf J, Adger WN, Lorenzoni I, Abrahamson V, Raine R. Social capital, individual responses to heat waves and climate change adaptation: an empirical study of two UK cities. Glob Environ Chang. 2010;20(1):44-52. https://doi.org/10.1016/j.gloenvcha.2009.09.004.

19. van Loenhout JAF, Rodriguez-Llanes JM, Guha-Sapir D. Stakeholders' perception on National Heatwave Plans and their local implementation in Belgium and the Netherlands. Int J Environ Res Public Health. 2016;13(11): 1120. https://doi.org/10.3390/ijerph13111120.

20. Bassil KL, Cole DC. Effectiveness of public health interventions in reducing morbidity and mortality during heat episodes: a structured review. Int J Environ Res Public Health. 2010;7(3):991-1001. https://doi.org/10.3390/ ijerph7030991.

21. Abrahamson V, Wolf J, Lorenzoni I, Fenn B, Kovats S, Wilkinson P, et al. Perceptions of heatwave risks to health: interview-based study of older people in London and Norwich, UK. J Public Health (Oxf). 2009;31(1):119-26.

22. Age UK. Advice for staying cool in a heatwave: Age UK; 2019 cited 2019. Available from: https://www.ageuk.org.uk/information-advice/healthwellbeing/mind-body/staying-cool-in-a-heatwave/.
23. Waldock KAM, Hayes M, Watt PW, Maxwell NS. Physiological and perceptual responses in the elderly to simulated daily living activities in UK summer climatic conditions. Public Health. 2018;161:163-70. https://doi.org/10.1016/j. puhe.2018.04.012.

\section{Publisher's Note}

Springer Nature remains neutral with regard to jurisdictional claims in published maps and institutional affiliations.

\section{Ready to submit your research? Choose BMC and benefit from:}

- fast, convenient online submission

- thorough peer review by experienced researchers in your field

- rapid publication on acceptance

- support for research data, including large and complex data types

- gold Open Access which fosters wider collaboration and increased citations

- maximum visibility for your research: over $100 \mathrm{M}$ website views per year

At BMC, research is always in progress.

Learn more biomedcentral.com/submissions 